\title{
Introduction to the Special Issue: Interplay of Family Factors and Cognitive-Affective Processes in Youth
}

\author{
Kristy Benoit Allen ${ }^{1} \cdot$ Patricia Z. Tan ${ }^{2,3} \cdot$ Ronald J. Prinz ${ }^{4}$ \\ Accepted: 15 February 2022 / Published online: 8 March 2022 \\ (c) The Author(s), under exclusive licence to Springer Science+Business Media, LLC, part of Springer Nature 2022
}

\begin{abstract}
Substantial research suggests that caregivers and families are powerful socialization agents when it comes to how youth process and regulate cognitive-affective information, which in turn can be a risk or resilience factor for various forms of developmental psychopathology. To this end, Clinical Child and Family Psychology Review features this special journal issue on the "Interplay of Family Factors \& Cognitive-Affective Processes in Youth." Featured articles review a wide array of methodologies and highlight numerous forms of cognitive-affective processing and family contextual factors. Multiple themes emerged across the twelve articles, emphasizing the need to examine (1) complex pathways within families, (2) the quality of cognitive-affective processes across individuals, (3) neurodevelopmental pathways linking socialization and cognitive-affective processes, (4) nuanced methods to assess "in-the-moment" cognitive-affective processes, (5) the impact of cultural background on how family factors intersect with youth cognitive-affective processes, and (6) the socialization of positive emotion. These papers showcase the applicability of this significant area of research for future efforts in prevention and intervention with youth at risk for, or already experiencing, some form of psychopathology.
\end{abstract}

Cognitive-affective processing in youth has been implicated in almost all forms of developmental psychopathology (Cicchetti et al., 1995; Ip et al., 2019; Izard et al., 2006). For example, aspects of cognitive-affective processing such as emotion reactivity and regulation, information processing biases, reward processing, stress reactivity, and subprocesses of cognitive control are thought to play important etiological roles in the development and maintenance of youth psychopathology (e.g., Kertz et al., 2016; Ladouceur et al., 2005; Nigg, 2017; White et al., 2011). A dimensional view of these cognitive-affective processes suggests that they are important for adaptive functioning, and when disrupted, also contribute to risk for negative developmental outcomes, such as psychiatric illness (Fernandez et al., 2016; McTeague et al.,

Kristy Benoit Allen

kallen51@utk.edu

1 Department of Psychology, University of Tennessee, Knoxville, USA

2 Department of Psychiatry and Biobehavioral Sciences, UCLA David Geffen School of Medicine, Los Angeles, USA

3 Department of Mental Health, VA Greater Los Angeles Healthcare System, Los Angeles, USA

4 University of South Carolina, Research Center for Child Well-Being, Columbia, USA
2016). Emerging research points to the powerful socialization effects that caregivers and families can have on how children process and regulate cognitive-affective information, serving as risk or protective processes in trajectories across childhood and adolescence (Morris et al., 2007). Facets of family contextual factors implicated include dyadic social interactions, parent-child coregulation, caregivers' responses to youth emotional expressions, parental cognitive-affective processing, emotion socialization, parenting behaviors, and parental psychopathology (e.g., Benoit Allen et al., 2015; Feldman \& Eidelman, 2004; Goodman, 2020; Milojevich et al., 2020; Pozzi et al., 2020; Tan et al., 2020).

To advance an understanding of how family factors and youth cognitive-affective processes might co-shape each other, we solicited conceptual and review articles on the interplay between biobehavioral processes underlying youth's cognitive-affective processing and family factors. We sought articles using diverse methodologies to examine this interplay, in order to look across levels of analysis and further our understanding of the complex, transactional pathways by which family context shapes the cognitive-affective processes contributing to developmental outcomes.

This special issue includes 12 articles that offer conceptual models and integrative reviews of research bearing on the powerful socialization effects families have on how 
children process and regulate cognitive-affective information. A number of critical themes emerged. First, multiple authors emphasize the importance of going beyond simple parent-to-child transmission models, noting how cognitiveaffective processes often influence the development of both child and caregiver. For example, in updating an influential model from over a decade ago, Aktar ("Intergenerational Transmission of Anxious Information Processing Biases: An Updated Conceptual Model") reviews studies showing that parenting behavior is not impacted only by parental anxiety and parent threat-related biases but also by child anxiety and child threat-related biases. Hovmand, Calzada, Gulbas, Kim, Chung, Kuhlberg, Huasmann-Stabile, and Zayas ("System Dynamics of Cognitive Vulnerabilities and Family Support Among Latina Children and Adolescents") go beyond bidirectionality, using a system dynamics approach that emphasizes feedback loops via which family responses to youth avoidant coping and maladaptive behaviors contribute to behavioral patterns that become more entrenched over time. Ratliff, Kerr, Cosgrove, Simmons, and Morris ("The Role of Neurobiological Bases of Dyadic Emotion Regulation in the Development of Psychopathology: CrossBrain Associations between Parents and Children") provide a conceptual framework wherein cross-brain associations impact the behavioral and physiological processes inherent in emotion regulation in both child and caregiver. And finally, Abraham and Feldman ("The Neural Basis of Human Fatherhood: A Unique Biocultural Perspective on Plasticity of Brain and Behavior") argue that the paternal brain changes in adaptive ways when fathers are actively involved in emotion socialization and changes which in turn support the development of emotion regulation abilities in offspring. Together, these articles highlight the unique and interactive risk and resilience effects caregivers and youth exert on each other. We hope this collection will help spur future research using study designs and analytical tools that allow for the testing of more sophisticated models.

A second theme to emerge is that the quality and characteristics of cognitive-affective processes should be considered across individuals, particularly within families. For example, Paley and Hajal ("Conceptualizing Emotion Regulation and Coregulation as Family Level Phenomena") argue that coregulation, or the processes by which caregivers provide support as children navigate their emotional experiences, should be studied as a family-level phenomenon rather than a dyadic process. Looking within dyads, Perlman, Lunkenheimer, Panlilio, and Pérez-Edgar ("Parent-to-Child Anxiety Transmission through Dyadic Social Dynamics: A Dynamic Developmental Model") suggest that the key mechanism explaining how anxiety transmits from parent to child is daily moment-to-moment interactions, and it is through this dyadic synchrony and emotion modeling that youth develop maladaptive cognitive-affective processing. Finally, the McKone and Silk article ("The Emotion Dynamics Conundrum in Developmental Psychopathology: Similarities, Distinctions, and Adaptiveness of Affective Variability and Socioaffective Flexibility") discusses the importance of considering both intrapersonal and interpersonal emotion dynamics and how these contribute to developmental trajectories toward health or disorder.

Three articles in this special issue sought to specify neurodevelopmental pathways linking caregiver socialization processes and the development of biological systems thought to underpin cognitive-affective processing. Kemp, Bernier, Lebel, and Kopala-Sibley ("Associations Between Parental Mood and Anxiety Psychopathology and Offspring Brain Structure: A Scoping Review") conclude from their review, despite mixed findings, that youth with a parental history of anxiety or mood disorders may show atypicalities in brain regions implicated in emotional processing, as well as differences in patterns of cortical gray matter thickening. The Bourne, Korom, and Dozier article ("Consequences of Inadequate Caregiving for Children's Attachment, Neurobiological Development, and Adaptive Functioning) reviews findings on the effects of disrupted attachment on children's neurophysiological processes and emotional behaviors. Finally, Ratliff, Kerr, Cosgrove, Simmons, and Morris ("The Role of Neurobiological Bases of Dyadic Emotion Regulation in the Development of Psychopathology: Cross-Brain Associations between Parents and Children") suggest that cross-brain connectivity may serve as the neurobiological basis for dyadic emotion regulation.

Several articles present arguments for the delineation of novel, nuanced methods for assessing "in-the-moment" cognitive-affective processes, suggesting that these methods might add to what has already been derived from more traditional assessment approaches. Stoop and Cole ("Listening In: An Alternative Method for Measuring the Family Emotional Environment") present the Electronically Activated Recorder (EAR) as a viable option for the objective measurement of the family emotional environment, which could add to an understanding of children's emotional development. McKone and Silk ("The Emotion Dynamics Conundrum in Developmental Psychopathology: Similarities, Distinctions, and Adaptiveness of Affective Variability and Socioaffective Flexibility") focus on in-the-moment intrapersonal and interpersonal emotion dynamics. Perlman, Lunkenheimer, Panlilio, and Pérez-Edgar ("Parent-to-Child Anxiety Transmission through Dyadic Social Dynamics: A Dynamic Developmental Model") suggest that the time scale of moment-to-moment coordination between parent and child may help generate new insights into developmental and clinical patterns.

Two articles address how issues of cultural diversity intersect with family factors and youth cognitive-affective processing to influence developmental trajectories. Doan, 
Yu, Wright, Fung, Saleem, \& Lau ("Resilience and Family Socialization Processes in Ethnic Minority Youth: Illuminating the Achievement-Health Paradox") highlight the importance of considering ecological context when outlining health-promoting vs. risk-enhancing socialization patterns, as well as underscore the costs of regulatory processes that have been defined as adaptative based on findings that have largely excluded families from marginalized communities. Noting that Latinx populations are not adequately represented in clinical trials research, Hovmand, Calzada, Gulbas, Kim, Chung, Kuhlberg, Huasmann-Stabile, and Zayas ("System Dynamics of Cognitive Vulnerabilities and Family Support Among Latina Children and Adolescents") draw on qualitative interview data from Latina youth to develop a formal feedback theory of risk for depression and suicidal ideation during school transitions.

Finally, the last article in this special issue, authored by Breaux, Lewis, Cash, Shroff, Burkhouse, and Kujawa ("Parent Emotion Socialization and Positive Emotions in Child and Adolescent Clinical Samples: A Systematic Review and Call to Action"), highlights a neglected area of study in the emotional socialization literature, namely positive emotion. Their review highlights links among positive affect, how positive affect is socialized by caregivers, and various clinical outcomes.

In this special issue, the many authors of these articles offer a wealth of information and ideas in the multi-faceted area of family factors and youth cognitive-affective processing. Many of the key variables and relationships are more prominent or important during specific developmental windows, and most authors directly address the clinical implications of their reviews and conceptual papers. We hope that future research in this area will deploy longitudinal designs to enable the testing of key relationships over multiple developmental periods. We anticipate that the kinds of applications exemplified in this special issue will broaden our knowledge of inter- and intraindividual processes to target for the prevention and intervention of negative developmental outcomes in youth.

Data Availability Data sharing is not applicable to this article as no datasets were generated or analysed during the current study.

\section{Declarations}

Conflict of interest All authors certify that they have no affiliations with or involvement in any organization or entity with any financial interest or non-financial interest in the subject matter or materials discussed in this manuscript.

Ethical approval As this article did not include the analysis of data, ethics approval was not required.

\section{References}

Benoit Allen, K., Silk, J. S., Meller, S., Tan, P.Z., Ladouceur, C. D., Sheeber, L. B., ... Ryan, N. D. (2015). Parental autonomy granting and child perceived control: Effects on the everyday emotional experience of anxious youth. Journal of Child Psychology and Psychiatry, 57(7), 835-842.

Cicchetti, D., Ackerman, B. P., \& Izard, C. E. (1995). Emotions and emotion regulation in developmental psychopathology. Development and Psychopathology, 7(1), 1-10.

Feldman, R., \& Eidelman, A. I. (2004). Parent-infant synchrony and the social-emotional development of triplets. Developmental Psychology, 40(6), 1133-1147.

Fernandez, K. C., Jazaieri, H., \& Gross, J. J. (2016). Emotion regulation: A transdiagnostic perspective on a new RDoC domain. Cognitive Therapy and Research, 40(3), 426-440.

Goodman, S. H. (2020). Intergenerational transmission of depression. Annual Review of Clinical Psychology, 16, 213-238.

Ip, K. I., Jester, J. M., Sameroff, A., \& Olson, S. L. (2019). Linking Research Domain Criteria (RDoC) constructs to developmental psychopathology: The role of self-regulation and emotion knowledge in the development of internalizing and externalizing growth trajectories from ages 3 to 10. Development and Psychopathology, 31(4), 1557-1574.

Izard, C. E., Youngstrom, E. A., Fine, S. E., Mostow, A. J., \& Trentacosta, C. J. (2006). Emotions and developmental psychopathology. In D. Cicchetti \& D. J. Cohen (Eds.), Developmental psychopathology: Theory and method (pp. 244-292). Wiley.

Kertz, S. J., Belden, A. C., Tillman, R., \& Luby, J. (2016). Cognitive control deficits in shifting and inhibition in preschool age children are associated with increased depression and anxiety over 7.5 years of development. Journal of Abnormal Child Psychology, 44(6), 1185-1196.

Ladouceur, C. D., Dahl, R. E., Williamson, D. E., Birmaher, B., Ryan, N. D., \& Casey, B. J. (2005). Altered emotional processing in pediatric anxiety, depression, and comorbid anxiety-depression. Journal of Abnormal Child Psychology, 33(2), 165-177.

McTeague, L. M., Goodkind, M. S., \& Etkin, A. (2016). Transdiagnostic impairment of cognitive control in mental illness. Journal of Psychiatric Research, 83, 37-46.

Milojevich, H. M., Machlin, L., \& Sheridan, M. A. (2020). Early adversity and children's emotion regulation: Differential roles of parent emotion regulation and adversity exposure. Development and Psychopathology, 32(5), 1788-1798.

Morris, A. S., Silk, J. S., Steinberg, L., Myers, S. S., \& Robinson, L. R. (2007). The role of the family context in the development of emotion regulation. Social Development, 16(2), 361-388.

Nigg, J. T. (2017). Annual research review: On the relations among self-regulation, self-control, executive functioning, effortful control, cognitive control, impulsivity, risk-taking, and inhibition for developmental psychopathology. Journal of Child Psychology and Psychiatry, 58(4), 361-383.

Pozzi, E., Simmons, J. G., Bousman, C. A., Vijayakumar, N., Bray, K. O., Dandash, O., ... Whittle, S. L. (2020). The influence of maternal parenting style on the neural correlates of emotion processing in children. Journal of the American Academy of Child \& Adolescent Psychiatry, 59(2), 274-282.

Tan, P. Z., Oppenheimer, C. W., Ladouceur, C. D., Butterfield, R. D., \& Silk, J. S. (2020). A review of associations between parental emotion socialization behaviors and the neural substrates of emotional reactivity and regulation in youth. Developmental Psychology, $56(3), 516$.

White, L. K., Suway, J. G., Pine, D. S., Bar-Haim, Y., \& Fox, N. A. (2011). Cascading effects: The influence of attention bias to 
threat on the interpretation of ambiguous information. Behaviour Research and Therapy, 49(4), 244-251.

Publisher's Note Springer Nature remains neutral with regard to jurisdictional claims in published maps and institutional affiliations. 